\title{
Colonic Web
}

National Cancer Institute

\section{Source}

National Cancer Institute. Colonic Web. NCI Thesaurus. Code C101315.

A pathologic fibrous net that impedes passage of intestinal contents through the colon. 